\title{
The expression and prognostic value of ischemia modified albumin (IMA), red blood cell distribution width (RDW), and lipoprotein (LP) in patients with diabetes mellitus complicated with coronary heart disease
}

\author{
Lu Xiang ${ }^{1,2 \#}$, Min Zhang ${ }^{2,3 \#}$, Hongying $\mathrm{Wu}^{1,2}$, Dili Xie ${ }^{2,4}$ \\ ${ }^{1}$ Department of Geriatric Medicine, Sichuan Provincial People's Hospital, University of Electronic Science and Technology of China, Chengdu, \\ China; ${ }^{2}$ Chinese Academy of Sciences, Sichuan Translational Medicine Research Hospital, Chengdu, China; ${ }^{3}$ Department of Outpatients, Sichuan \\ Provincial People's Hospital, University of Electronic Science and Technology of China, Chengdu, China; ${ }^{4}$ Department of Geriatric Cardiology, \\ Sichuan Provincial People's Hospital, University of Electronic Science and Technology of China, Chengdu, China \\ Contributions: (I) Conception and design: L Xiang, M Zhang; (II) Administrative support: H Wu, D Xie; (III) Provision of study materials or \\ patients: L Xiang, M Zhang; (IV) Collection and assembly of data: L Xiang, M Zhang; (V) Data analysis and interpretation: L Xiang, M Zhang; \\ (VI) Manuscript writing: All authors; (VII) Final approval of manuscript: All authors. \\ "These authors contributed equally to this work. \\ Correspondence to: Dili Xie. Department of Geriatric Cardiology, No.32 West Section 2, First Ring Road, Chengdu 610072, China. \\ Email: xie1981dili@163.com; Hongying Wu. Department of Geriatric Medicine, No.32 West Section 2, First Ring Road, Chengdu 610072, China. \\ Email: why17708130@163.com.
}

Background This study explored the levels and prognostic value of ischemia modified albumin (IMA), red blood cell distribution width (RDW), and lipoprotein (LP) in patients with diabetes melltus (DM) complicated withcoronary heart disease (CHD).

Methods: A total of 95 patients with DM who were diagnosed and treatedfrom January 2018 to January 2019 were retrospectively analyzed. All included patients underwent percutaneous coronary intervention (PCI). Patients with DM complicated with CHDwere designated group A $(n=61)$ and patients without CHD complications were designated group B ( $n=34)$. During the same period, 45 patients without DM who underwent physical examination in our hospital were included as a control group. The levels of IMA, LP, and RDW in the 3 groups of patients were compared. The study examined the occurrence of cardiovascular events after PCI treatment in patients with DM complicated with CHD, and the related risk factors were assessed using multivariate logistic regression analysis. Furthermore, the receiver operating characteristic (ROC) curve was used to analyze the value of IMA, LP, and RDW in predicting cardiovascular events in DM patients complicated with CHD.

Results: The levels of IMA, LP, and RDW werehigher in groups A and B compared to the control group $(\mathrm{P}<0.05)$. In patients who experienced a cardiovascular event, the levels of IMA, LP, and RDW were higher than those observed in patients who did not experience a cardiovascular event $(\mathrm{P}<0.05)$. The area under the ROC curve of IMA, LP, RDW, and the combination of the three factors for the prediction of cardiovascular events were $0.910,0.774,0.846$, and 0.995 , respectively. The combined detection of the 3 factors showed the best predictive value.Patients with abnormal values in blood lipids, blood creatinine, IMA, LP, and RDW had a significantly poorer prognosis in terms of adverse cardiovascular events $(\mathrm{P}<0.05)$. The unconditional multivariate logistic regression model showed that abnormal levels of blood creatinine, IMA, LP, and RDW were independent risk factors for cardiovascular events in patients with DM complicated with CHD after PCI treatment $(\mathrm{P}<0.05)$.

Conclusions: The levels of IMA, RDW, and LP wereincreased in patients with DM complicated with CHD. Furthermore, abnormal levels of IMA, LP, and RDW are independent risk factors that affect cardiovascular events in these patients following PCI treatment. The combined detection of all three 
indicators may be an effective means to predict the prognosis of these patients.

Keywords: Ischemia modified albumin (IMA); red blood cell distribution width (RDW); lipoprotein (LP); diabetes mellitus complicated with coronary heart disease (CHD); percutaneous coronary intervention treatment (PCI treatment)

Submitted Jan 29, 2021. Accepted for publication Apr 04, 2021.

doi: 10.21037/apm-21-425

View this article at: http://dx.doi.org/10.21037/apm-21-425

\section{Introduction}

Diabetes melltus (DM) is a group of lifelong metabolic diseases characterized by chronic blood glucose elevation which can be caused by multiple factors. Oxidative stress resulting from long-term hyperglycemia can aggravate arteriosclerosis and cause various complications (1). Coronary heart disease (CHD) is a significant complication for diabetic patients with a high-risk of death. With the increasing incidence of diabetic patients complicated with $\mathrm{CHD}$, the search for prognostic biochemical indicators with significant sensitivity and specificity has become a major clinical focus.

Ischemia modified albumin (IMA) is a novel and effective ischemic marker (2). Many studies have shown that IMA can sensitively reflect myocardial ischemia and can be used in the early diagnosis of acute coronary syndromes. This has great significance for patient risk stratification and treatment guidance (3). Malondialdehyde and advanced oxidation protein products can be considered better than IMA in the evaluation of diabetes progression, but malondialdehyde is more useful as a diagnostic indicator to detect vascular complications. Hemoglobin measurement is of greater value than the oxidative stress markers in the prediction of vascular complications (4). The red blood cell distribution width (RDW) is a parameter that reflects the physical heterogeneity of red blood cells. Recently, studies have revealed that RDW can be used for early risk stratification of CHD patients and is an important predictor for acute cardiovascular events (5). RDW values are found to be increased in the diabetic coronary artery disease population. Higher RDW values are related to more extensive and complex coronary lesions in patients with DM (6). Diabetic patients with high-coronary artery calcium scores and significant coronary artery disease have higher RDW (7). Elevated serum lipoprotein is a significant risk factor; and the risk of $\mathrm{CHD}$ appears to increase with increasing serum lipoprotein concentrations (8). Lipoproteins (LPs) are spherical particles composed of a hydrophobic core rich in sterol lipids and triglycerides, and a shell composed of proteins, phospholipids, and cholesterol (9). LPs play an important role in the packaging and storage of extracellular lipids as well as in molecular transportation and metabolism. It has been shown that abnormal LP metabolism is closely related to the occurrence of diabetes, arteriosclerosis, and other diseases (10). Both low-molecular-weight lipoprotein and low relative lymphocyte concentration are significant and independent risk factors for coronary heart disease and that the combination thereof more strongly predicts coronary heart disease in patients with type 2 diabetes mellitus (11). Glycation of high-density lipoprotein exerts a deleterious effect on the development of cardiovascular complications in diabetes (12). Therefore, this study investigated the levels and prognostic value of IMA and LPs, and the RDW in patients with DM complicated with CHD with the aim of providing an effective biomarker for clinical application. We present the following article in accordance with the STARD reporting checklist (available at http://dx.doi.org/10.21037/apm-21-425).

\section{Methods}

\section{General information}

A total of 95 patients with DM who were diagnosed and treated in our hospital from January 2018 to January 2019 were retrospectively studied. There were 61 patients with DM complicated with CHD (designated group A) and 34 patients with DM and no CHD complications (designated group B). All patientsagreed to participate in this study and signed an informed consent form. The study was conducted in accordance with the Declaration of Helsinki (as revised in 2013). This study was approved by the Sichuan Provincial People's Hospital, University of Electronic Science and 
Table 1 A comparison of the general patient characteristics

\begin{tabular}{|c|c|c|c|c|c|c|}
\hline Groups & $\begin{array}{c}\text { Gender } \\
\text { (male/female) }\end{array}$ & Age (year) & $\begin{array}{l}\text { Residence } \\
\text { (city/town) }\end{array}$ & $\begin{array}{l}\text { BMI (body mass } \\
\text { index, } \mathrm{kg} / \mathrm{m}^{2} \text { ) }\end{array}$ & $\begin{array}{l}\text { History of smoking } \\
\text { (yes/no) }\end{array}$ & $\begin{array}{l}\text { History of hypertension } \\
\text { (yes/no) }\end{array}$ \\
\hline Group A ( $n=61)$ & $31 / 30$ & $54.11 \pm 5.13$ & $32 / 29$ & $25.11 \pm 3.65$ & $32 / 29$ & $33 / 28$ \\
\hline Control group $(n=45)$ & $23 / 22$ & $53.79 \pm 5.26$ & $26 / 19$ & $24.97 \pm 3.59$ & $24 / 21$ & $27 / 18$ \\
\hline$\chi^{2} / F$ & 0.042 & 0.20 & 0.818 & 0.15 & 0.057 & 0.420 \\
\hline
\end{tabular}

BMI, body mass index.

Technology of China (No. 20171212). The inclusion criteria were as follows: (I) patients diagnosed with DM according to the criteria of the Chinese Guidelines for Diabetes Prevention and Treatment (13); and (II) patients with CHD confirmed by coronary angiography showing greater than $50 \%$ coronary artery stenosis. The following patients were excluded from the study: (I) patients with severe or active infectious diseases; (II) patients with insufficiencies of the liver and/or kidneys; (III) patients with malignant tumors; and (IV) patients with severe hematological diseases. A total of 45 patients without DM who underwent physical examination in our hospital during the same period were included as the control group. All relevant clinical data were true and complete for all study subjects, and there were no differences in the general patient characteristics such as age, gender, and body mass index (BMI) among the three groups (Table 1).

\section{Study method}

All patients with DM complicated with CHD underwent percutaneous coronary intervention (PCI), and the levels of IMA, LP, and RDW were compared among the three groups. The occurrence of cardiovascular events in DM patients complicated with CHD after PCI treatment was analyzed. The receiver operating characteristic (ROC) curve was used to analyze the predictive values of IMA, LP, and RDW for cardiovascular events in DM patients complicated with CHD. Multivariate logistic regression analysis was used to assess the risk factors associated with cardiovascular events in DM patients complicated with CHD after PCI treatment.

The cardiovascular events defined in this study included hypotension during hospitalization, malignant arrhythmia, cardiac function Killip $\geq$ III, cardiogenic death, and recurrent myocardial infarction.

\section{Observation indicators}

(I) IMA test: at admission, $5 \mathrm{~mL}$ of venous blood was collected and centrifuged at 1,000 rpm for 10 minutes. The supernatant $(400 \mu \mathrm{L})$ was collected and the IMA content was measured using an enzyme-linked immunosorbent assay (ELISA) kit according to the manufacturer's instructions (Cusabio, Maryland, USA). An IMA level $\leq 65 \mathrm{U} / \mathrm{mL}$ was defined as a normal value.

(II) LP test: fasting venous blood $(5 \mathrm{~mL})$ was collected from all patients on the morning of the second day after admission (the day of the physical examination). The blood was centrifuged to separate the serum and LP levels were assessed using an immunoturbidimetric kit (Shanghai Zhicheng Biotechnology Co., Ltd., Shanghai, China) and the Roche ModularP800 automatic biochemical analyzer. A LP measurement of $<300 \mathrm{mg} / \mathrm{L}$ was considered a normal value.

(III) RDW test: fasting venous blood $(5 \mathrm{~mL})$ was collected from all patients on the morning of the second day after admission and the RDW was determined using an automatic blood cell analyzer. A RDW range between $11 \%$ and $16 \%$ was considered normal.

\section{Statistical analysis}

SPSS 20.0 statistical software was used for data analysis. Measurement data were expressed as mean \pm standard deviation $(\bar{x} \pm \mathrm{s})$ and assessed using the $\mathrm{F} / \mathrm{t}$-test. Counting data were expressed in percentages (\%) and the $\chi^{2}$ test was used for comparison. The ROC curve was used to analyze the predictive value of IMA, LP, and RDW for 
Table 2 A comparison of the levels of ischemia modified albumin, lipoprotein, and red blood cell distribution width in the 3 groups of patients $(\bar{x} \pm \mathrm{S})$

\begin{tabular}{lccc}
\hline Groups & LP $(\mathrm{mg} / \mathrm{L})$ & $\mathrm{IMA}(\mathrm{U} / \mathrm{mL})$ & $\mathrm{RDW}(\%)$ \\
\hline Group A $(\mathrm{n}=61)$ & $399.83 \pm 40.39^{\star \#}$ & $95.41 \pm 5.63^{\star \#}$ & $24.39 \pm 5.71^{\star \#}$ \\
Group B $(\mathrm{n}=34)$ & $321.35 \pm 31.58^{*}$ & $77.95 \pm 3.81^{*}$ & $22.51 \pm 5.16^{*}$ \\
Control group $(\mathrm{n}=45)$ & $153.63 \pm 28.35$ & $20.11 \pm 4.69$ & $10.82 \pm 5.53$ \\
F & 644.67 & 3114.44 & 84.49 \\
P & $<0.001$ & $<0.001$ & $<0.001$ \\
\hline
\end{tabular}

*, compared with the control group, $\mathrm{P}<0.05$; ", compared with group B, P<0.05. LP, lipoprotein; IMA, ischemia modified albumin; RDW, red blood cell distribution width.

Table 3 A comparison of the levels of ischemia modified albumin, lipoprotein, and red blood cell distribution width in patients with and without an adverse cardiovascular event $(\bar{x} \pm S)$

\begin{tabular}{lccc}
\hline Groups & LP $(\mathrm{mg} / \mathrm{L})$ & $\mathrm{IMA}(\mathrm{U} / \mathrm{mL})$ & $\mathrm{RDW}(\%)$ \\
\hline Cardiovascular event group $(\mathrm{n}=28)$ & $378.13 \pm 25.39$ & $83.63 \pm 11.23$ & $23.63 \pm 6.35$ \\
Non-cardiovascular event group $(\mathrm{n}=33)$ & $151.37 \pm 20.71$ & $25.63 \pm 9.67$ & $9.37 \pm 5.17$ \\
$t$ & 38.421 & 21.678 & 9.669 \\
$\mathrm{P}$ & $<0.001$ & $<0.001$ & $<0.001$ \\
\hline
\end{tabular}

LP, lipoprotein; IMA, ischemia modified albumin; RDW, red blood cell distribution width.

cardiovascular events in patients with DM complicated by CHD. The relevant factors affecting the incidence of cardiovascular events were analyzed by logistic regression and $\alpha=0.05$ was considered significant.

\section{Results}

\section{The levels of IMA, LP, and RDW in the three groups of} patients

The levels of IMA, LP, and RDW were significantly higher in groups $\mathrm{A}$ and $\mathrm{B}$ compared to the control group $(\mathrm{P}<0.05)$, with the levels significantly higher in group A compared to group $\mathrm{B}(\mathrm{P}<0.05$; Table 2$)$.

The occurrence of cardiovascular events in diabetes mellitus patients complicated with chronic heart disease after PCI treatment

A total of 28 cardiovascular events occurred in $61 \mathrm{DM}$ patients complicated with CHD after PCI treatment, that is an incidence rate of $45.90 \%$. The adverse cardiovascular events included 9 cases of hypotension during hospitalization, 10 cases of malignant arrhythmia, 4 cases of cardiac function Killip $\geq \mathrm{III}, 2$ cases of cardiac deaths, and
3 cases of recurrent myocardial infarction.

The predictive value of IMA, LP, and RDW for cardiovascular events in diabetes mellitus patients complicated by chronic heart disease after PCI treatment

The levels of IMA, LP, and RDW were higher in patients who suffered a cardiovascular event compared to patients in the non-cardiovascular event group $(\mathrm{P}<0.05$; Table 3).

The ROC curve was used to analyze the predictive values of IMA, LP, RDW, and the combined detection of all three factors on the occurrence of cardiovascular events in DM patients complicated by CHD after PCI treatment. The area under the curve for each predictive indicator and their combined detection was $0.910,0.774,0.846$, and 0.995 , respectively, where the combined detection achieved the best predictive performance (Figure 1).

\section{Single-factor analysis of the cardiovascular events in diabetes mellitus patients complicated by chronic heart disease after PCI treatment}

Gender, age, BMI, history of hypertension, and history of smoking did not significantly affect the incidence of 


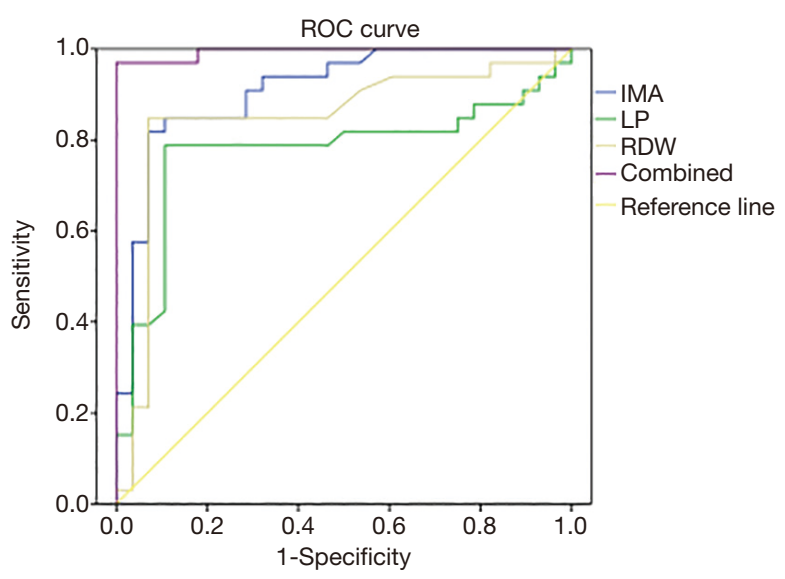

Figure 1 A comparison of the area under the curve for the prediction of cardiovascular events in diabetes mellitus patients complicated with coronary heart disease. The predictive values of IMA, LP, RDWand the combination of all three factors is shown. IMA, ischemia modified albumin; LP, lipoprotein; RDW, red blood cell distribution width; ROC, receiver operating characteristic.

cardiovascular events $(\mathrm{P}>0.05)$. However, patients with an abnormal level of blood lipids, blood creatinine, IMA, LP, or RDW showed a significantly poorer prognosis, with increased incidences of cardiovascular events $(\mathrm{P}<0.05$; Table 4).

\section{An analysis of the factors affecting cardiovascular events in diabetes mellitus patients complicated by chronic beart disease after PCI treatment}

Analyses with unconditional multivariate logistic regression models revealed that abnormal levels of blood creatinine, IMA, LP, and RDW were all independent risk factors for cardiovascular events in DM patients complicated with CHD after $\mathrm{PCI}$ treatment $(\mathrm{P}<0.05$; Table 5$)$.

\section{Discussion}

Abnormal glucose metabolism increases the morbidity and mortality of cardiovascular diseases. In fact, $46 \%$ of all newly diagnosed DM patients are complicated with CHD (14) and the National Cholesterol Education Program III considers DM and CHD to be "equal risk diseases" (15). PCI treatment is one of the mainstream methods for patients with DM complicated with CHD and is widely used in clinical practice. However, such patients often have severe coronary atherosclerosis and calcification, which can impede the efficacy of clinical treatments. Therefore, it is of great importance to target both the prevention and treatment of $\mathrm{DM}$ and CHD.

\section{The levels and prognostic value of IMA in diabetes mellitus patients complicated by chronic beart disease}

At present, the early diagnosis of CHD mainly relies on medical history, echocardiography, and coronary computed tomography (CT). However, the inclusion of these factors for the early prediction of vascular disease lacks sensitivity and specificity (16). IMA is formed when the body undergoes ischemia and the amino acid sequence of serum albumin is destroyed by free radicals and other substances resulting in changes in the binding capacity of albumin with transition metals. Thus, IMA has become a novel marker of myocardial ischemia $(2,17)$. Previous studies compared the IMA levels in DM patients without CHD, DM patients with stable angina, and DM patients with acute CHD. They found that the IMA levels were highest in DM patients with acute CHD, suggesting that human serum IMA is an important biochemical indicator for the early diagnosis of clinical CHD, and can be used to detect and evaluate the severity of disease, as well as for prognosis and treatment $(18,19)$. These results support the findings of our current study.

Further analysis of the risk factors that affect the occurrence of cardiovascular events in patients with DM complicated with CHD after PCI treatment found that abnormal IMA levels is one of the high-risk factors, suggesting that elevated IMA levels are closely related to the occurrence of cardiovascular events. Therefore, clinical management should focus on serum IMA testing in patients with DM complicated with CHD to accurately evaluate the progress of the disease and improve prognosis for patients.

\section{The levels and prognostic value of RDW in diabetes mellitus patients complicated by chronic beart disease}

Metabolic diseases generally involve the chronic inflammation process, and patients with DM typically have higher levels of chronic inflammation and oxidative stress (19). RDW can reflect the uniformity of red blood cells and is often used in the differential diagnosis of anemia. Recent studies have found that high levels of RDW can reflect potential inflammatory conditions. Some studies suggested that inflammatory responses can increase RDW by weakening iron metabolism, inhibiting the survival of erythropoietin, and shortening 
Table 4 Single-factor analysis of cardiovascular events in diabetes mellitus patients complicated by chronic heart disease after percutaneous coronary intervention treatment

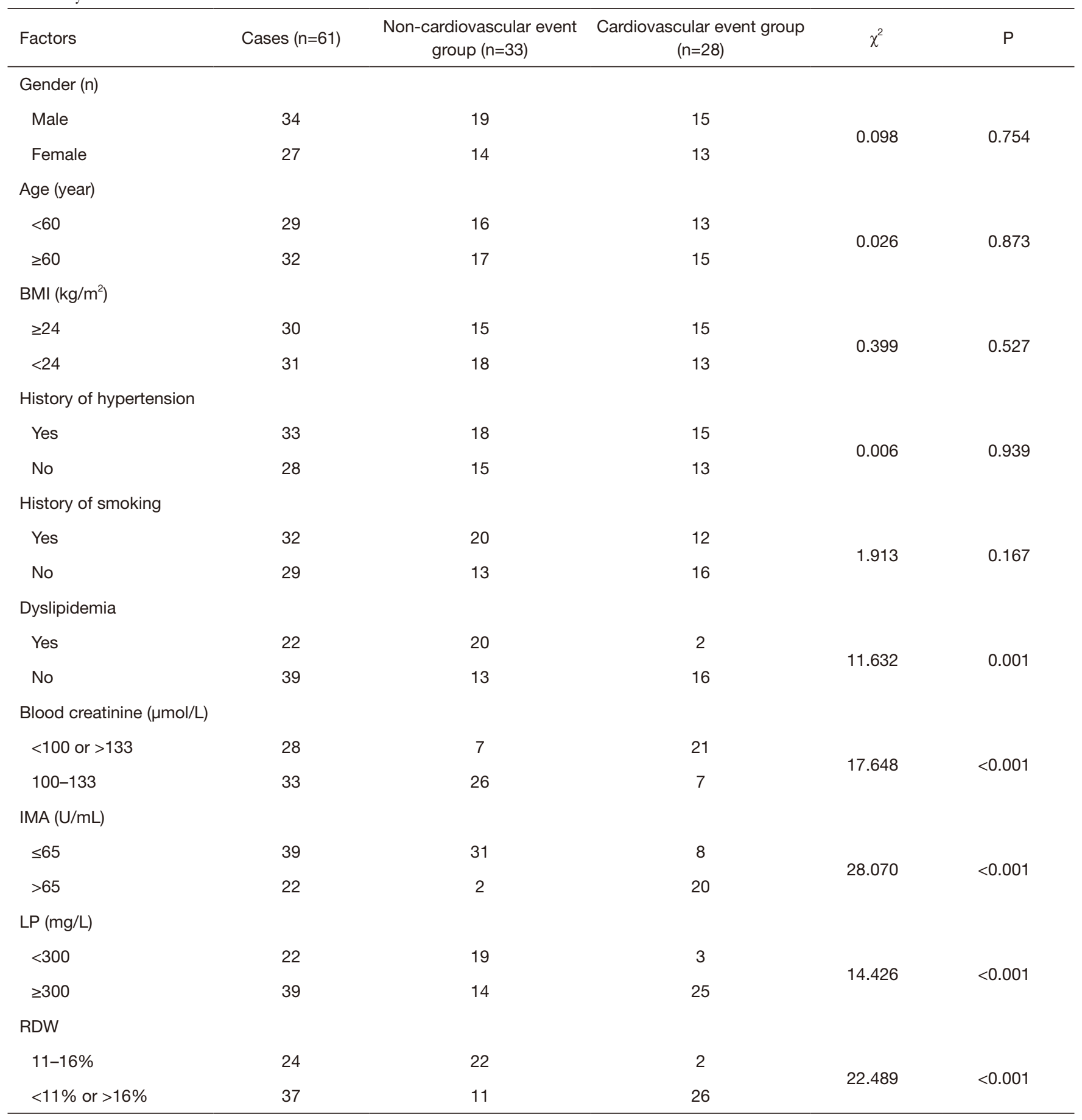

BMI, body mass index; LP, lipoprotein; IMA, ischemia modified albumin; RDW, red blood cell distribution width.

the lifespan of red blood cells $(20,21)$. In DM patients, the $\mathrm{RDW}$ level is related to the occurrence of microvascular and macrovascular diseases and is one of the clinical indicators for evaluating vascular complications. The results of this study demosntrated that RDW levels were significantly higher in patients with DM complicated with CHD and in 
Table 5 Multi-factorial analysis of cardiovascular events in diabetes mellitus patients complicated by chronic heart disease after percutaneous coronary intervention treatment

\begin{tabular}{lccccc}
\hline Factors & Regression coefficients & Standard error & Wald $\chi^{2}$ & $\mathrm{P}$ & OR (95\% Cl) \\
\hline Dyslipidemia (control group: normal) & 0.745 & 0.677 & 4.689 & 0.695 & $2.11(0.56-7.94)$ \\
$\begin{array}{l}\text { Blood creatinine }<100 \text { or }>133 \mu \mathrm{mol} / \mathrm{L} \\
\text { (control group: } 100-133 \text { ) }\end{array}$ & 0.866 & 0.181 & 4.468 & $<0.001$ & $2.38(1.67-3.39)$ \\
$\mathrm{IMA}>65 \mathrm{U} / \mathrm{mL}$ (control group: $\leq 65)$ & 0.795 & 0.143 & 4.433 & $<0.001$ & $2.21(1.67-2.93)$ \\
$\mathrm{LP} \geq 300 \mathrm{mg} / \mathrm{L}$ (control group: $<300$ ) & 0.894 & 0.135 & 4.469 & $<0.001$ & $2.44(1.88-3.19)$ \\
RDW $<11 \%$ or $>16 \%$ (control group: $11-16 \%)$ & 0.774 & 0.129 & 4.414 & $<0.001$ & $2.17(1.68-2.79)$ \\
\hline
\end{tabular}

IMA, ischemia modified albumin; LP, lipoprotein; RDW, red blood cell distribution width; OR, odds ratio; Cl, confidence interval.

patients with poor prognosis after treatment $(\mathrm{P}<0.05)$, which is consistent with previous studies (22). RDW values are found to be increased in the diabetic coronary artery disease population. Higher RDW values are related to more extensive and complex coronary lesions in patients with DM (6). Diabetic patients with high-coronary artery calcium scores and significant coronary artery disease have higher RDW (7). Elevated serum lipoprotein is a significant risk factor; and the risk of CHD appears to increase with increasing serum lipoprotein concentrations (8). These results suggested that a high RDW value is positively correlated with coronary artery disease, and can be used as a predictor for vascular complications in patients with DM. Since RDW is easy to detect, economical, and repeatable, it is of high value in the clinical setting.

Furthermore, the results of the unconditional multivariate logistic regression analysis revealed that abnormal levels of RDW affected the occurrence of cardiovascular events in patients with DM complicated by CHD after PCI treatment $(\mathrm{P}<0.05)$. This suggested that RDW may be useful in assessing the prognosis of patients with DM complicated by CHD. It is possible that the increase in RDW reduces the deformability of red blood cells, resulting in obstruction of the microcirculation. This can lead to an increase in peripheral blood resistance which is related to the severity of coronary artery disease $(23,24)$.

\section{The levels and prognostic value of LP in diabetes mellitus patients complicated by chronic heart disease}

LPs are composed of proteins and lipids, and can cause thrombosis and arteriosclerosis. LPs can cause atherosclerosis via several different mechanisms including: (I) inhibition of the tissue plasminogen activator; (II) enhancing the inhibition of plasmin by $\alpha 2$-antiplasmin; (III) competing with plasmin for binding to fibrin although it has no plasmin-like activity; (IV) enhancing the adhesion between monocytes and endothelial cells; and (V) binding to the plasminogen receptor on endothelial cells and promoting the transformation of macrophages into foam cells (25-27). Both lowmolecular-weight lipoprotein and low relative lymphocyte concentration are significant and independent risk factors for coronary heart disease and that the combination thereof more strongly predicts coronary heart disease in patients with type 2 diabetes mellitus (11). Glycation of high-density lipoprotein exerts a deleterious effect on the development of cardiovascular complications in diabetes (12). This current study demonstrated that DM patients complicated with CHD had significantly higher levels of LP compared to patients without CHD and healthy people $(\mathrm{P}<0.05)$. The multi-factor analysis showed that abnormal LP levels can affect the occurrence of cardiovascular events in DM patients complicated with CHD after PCI treatment $(\mathrm{P}<0.05)$, suggesting that LP levels can be used to assess the severity of CHD complicated with DM, and guide prognosis and treatment. In addition, the ROC curve showed that the combined detection of IMA, LP, and RDW achieved the highest predictive value for the occurrence of cardiovascular events in DM patients complicated by CHD after PCI treatment. This is consistent with previous studies (28). Therefore, the combined detection of IMA, LP and RDW can improve the predictive value of cardiovascular events in DM patients complicated with CHD after PCI treatment.

The unconditional multivariate logistic regression analysis showed that an abnormal level of blood creatinine was a risk factor for the occurrence of cardiovascular events in DM patients complicated with CHD after PCI treatment $(\mathrm{P}<0.05)$. Serum levels of creatinine can reflect glomerular filtration capacity, and abnormal renal function is often associated with a more serious state of arteriosclerosis (29). 
Therefore, DM patients complicated with CHD may need to control blood creatinine levels and repair renal function prior to PCI treatment to improve the clinical outcome.

In summary, the levels of IMA, RDW, and LP are significantly elevated in DM patients complicated with CHD. Abnormal levels of IMA, LP, and RDW are independent risk factors that affect cardiovascular events in DM patients complicated with CHD after PCI treatment and the combined detection of all three indicators may be an effective means for predicting the prognosis of such patients.

\section{Acknowledgments}

Funding: None.

\section{Footnote}

Reporting Checklist: The authors have completed the STARD reporting checklist. Available at http://dx.doi.org/10.21037/ apm-21-425

Data Sharing Statement: Available at http://dx.doi. org/10.21037/apm-21-425

Conflicts of Interest: All authors have completed the ICMJE uniform disclosure form (available at http://dx.doi. org/10.21037/apm-21-425). The authors have no conflicts of interest to declare.

Ethical Statement: The authors are accountable for all aspects of the work in ensuring that questions related to the accuracy or integrity of any part of the work are appropriately investigated and resolved. This study was approved by the Sichuan Provincial People's Hospital, University of Electronic Science and Technology of China (No. 20171212). The study was conducted in accordance with the Declaration of Helsinki (as revised in 2013). All patients agreed to participate in this study and signed an informed consent form.

Open Access Statement: This is an Open Access article distributed in accordance with the Creative Commons Attribution-NonCommercial-NoDerivs 4.0 International License (CC BY-NC-ND 4.0), which permits the noncommercial replication and distribution of the article with the strict proviso that no changes or edits are made and the original work is properly cited (including links to both the formal publication through the relevant DOI and the license). See: https://creativecommons.org/licenses/by-nc-nd/4.0/.

\section{References}

1. Huo X, Krumholz HM, Bai X, et al. Effects of Mobile Text Messaging on Glycemic Control in Patients With Coronary Heart Disease and Diabetes Mellitus: A Randomized Clinical Trial. Circ Cardiovasc Qual Outcomes 2019;12:e005805.

2. Yarcı Gursoy A, Caglar GS, Demirtas S. Ischemia modified albumin in perinatology. Eur J Obstet Gynecol Reprod Biol 2017;210:182-8.

3. Kawada T. Smoking and coronary heart disease in patients with type 2 diabetes mellitus. Diabetes Res Clin Pract 2017;133:211.

4. D'Souza JM, D'Souza RP, Vijin VF, et al. High predictive ability of glycated hemoglobin on comparison with oxidative stress markers in assessment of chronic vascular complications in type 2 diabetes mellitus. Scand J Clin Lab Invest 2016;76:51-7.

5. Li N, Zhou H, Tang Q. Red Blood Cell Distribution Width: A Novel Predictive Indicator for Cardiovascular and Cerebrovascular Diseases. Dis Markers 2017;2017:7089493.

6. Celik A, Karayakali M, Altunkas F, et al. Red cell distribution width is correlated with extensive coronary artery disease in patients with diabetes mellitus. Cardiovasc J Afr 2017;28:319-23.

7. Khalil A, Shehata M, Abdeltawab A, et al. Red blood cell distribution width and coronary artery disease severity in diabetic patients. Future Cardiol 2019;15:355-66.

8. Murase T, Okubo M, Amemiya-Kudo M, et al. Impact of elevated serum lipoprotein (a) concentrations on the risk of coronary heart disease in patients with type 2 diabetes mellitus. Metabolism 2008;57:791-5.

9. Hooda Y, Shin HE, Bateman TJ, et al. Neisserial surface lipoproteins: structure, function and biogenesis. Pathog Dis 2017. doi: $10.1093 / \mathrm{femspd} / \mathrm{ft} \times 010$.

10. Hammons L, Filopei J, Steiger D, et al. A narrative review of red blood cell distribution width as a marker for pulmonary embolism. J Thromb Thrombolysis 2019;48:638-47.

11. Suzuki T, Futami-Suda S, Igari Y, et al. Low-molecularweight lipoprotein (a) and low relative lymphocyte concentration are significant and independent risk factors for coronary heart disease in patients with type 2 diabetes mellitus: $\mathrm{Lp}(\mathrm{a})$ phenotype, lymphocyte, and coronary heart 
disease. Lipids Health Dis 2013;12:31.

12. Sun JT, Shen Y, Lü AK, et al. Glycation of high-density lipoprotein in type 2 diabetes mellitus. Chin Med J (Engl) 2013;126:4162-5.

13. Karaşin SS, Çift T. The Role of Ischemia-modified Albumin as a Biomarker in Preeclampsia. Rev Bras Ginecol Obstet 2020;42:133-9.

14. Goyal H, Lippi G, Gjymishka A, et al. Prognostic significance of red blood cell distribution width in gastrointestinal disorders. World J Gastroenterol 2017;23:4879-91.

15. Ahotupa M. Oxidized lipoprotein lipids and atherosclerosis. Free Radic Res 2017;51:439-47.

16. Hu ZD, Lippi G, Montagnana M. Diagnostic and prognostic value of red blood cell distribution width in sepsis: A narrative review. Clin Biochem 2020;77:1-6.

17. Rana JS, Liu JY, Moffet HH, et al. Ethnic Differences in Risk of Coronary Heart Disease in a Large Contemporary Population. Am J Prev Med 2016;50:637-41.

18. Coverdale JPC, Katundu KGH, Sobczak AIS, et al. Ischemia-modified albumin: Crosstalk between fatty acid and cobalt binding. Prostaglandins Leukot Essent Fatty Acids 2018;135:147-57.

19. Oran I, Oran B. Ischemia-Modified Albumin as a Marker of Acute Coronary Syndrome: The Case for Revising the Concept of "N-Terminal Modification" to "Fatty Acid Occupation" of Albumin. Dis Markers 2017;2017:5692583.

20. Salvagno GL, Sanchis-Gomar F, Picanza A, et al. Red blood cell distribution width: A simple parameter with multiple clinical applications. Crit Rev Clin Lab Sci 2015;52:86-105.

Cite this article as: Xiang $\mathrm{L}$, Zhang $\mathrm{M}, \mathrm{Wu} \mathrm{H}, \mathrm{Xie} \mathrm{D}$. The expression and prognostic value of ischemia modified albumin (IMA), red blood cell distribution width (RDW), and lipoprotein (LP) in patients with diabetes mellitus complicated with coronary heart disease. Ann Palliat Med 2021;10(4):44634471. doi: 10.21037/apm-21-425
21. Vural Yilmaz Z, Gencosmanoglu Turkmen G, Daglar K, et al. Elevated red blood cell distribution width is associated with intrahepatic cholestasis of pregnancy. Ginekol Pol 2017;88:75-80.

22. Silva Litao MK, Kamat D. Back to Basics: Red Blood Cell Distribution Width: Clinical Use beyond Hematology. Pediatr Rev 2018;39:204-9.

23. Rader DJ. Mediterranean Approach to Improving High-Density Lipoprotein Function. Circulation 2017;135:644-7.

24. Pan L, Segrest JP. Computational studies of plasma lipoprotein lipids. Biochim Biophys Acta 2016;1858:2401-20.

25. Jomard A, Osto E. Metabolism and Function of HighDensity Lipoproteins (HDL). Praxis (Bern 1994) 2019;108:477-86.

26. Lutomski CA, Gordon SM, Remaley AT, et al. Resolution of Lipoprotein Subclasses by Charge Detection Mass Spectrometry. Anal Chem 2018;90:6353-6.

27. Korkmaz MF, Tutanç M. Red blood cell distribution width as a useful marker for severity in pediatric acute gastroenteritis. Pediatr Int 2019;61:1109-13.

28. Wei S, Cui H, Zhang S, et al. Red Blood Cell Distribution Width Predicts Postoperative Death of Infective Endocarditis. Int Heart J 2020;61:524-30.

29. Zhu YT, Xiong J, Guo W, et al. The effects of different hydration strategies before endovascular treatments to the renal function of the arteriosclerosis obliterans patients. Zhonghua Yi Xue Za Zhi 2010;90:1155-8.

(English Language Editor: J. Teoh) 